\title{
Contribuições da Teoria da Desintegração Positiva para a Área de Superdotação
}

\author{
Juliana Célia de Oliveira ${ }^{1}$ \\ Altemir José Gonçalves Barbosa \\ Universidade Federal de Juiz de Fora \\ Eunice M. L. Soriano de Alencar \\ Universidade de Brasília
}

\begin{abstract}
RESUMO - A Teoria da Desintegração Positiva (TDP), proposta por Dabrowski, constitui importante ferramenta para a compreensão do desenvolvimento emocional de superdotados. Trata-se de uma teoria do desenvolvimento da personalidade que enfatiza o papel desempenhado pelas emoções no potencial de desenvolvimento humano. O objetivo deste artigo é apresentar conceitos centrais da TDP e suas contribuições para a área da superdotação. É enfatizado o conceito de sobre-excitabilidade, que, além de ser considerado um indicador de superdotação, representa um construto chave para o desenvolvimento de medidas e para promoção de intervenções que tenham como foco as características de personalidade e emocionais dos superdotados.
\end{abstract}

Palavras-chave: Teoria da Desintegração Positiva, Dabrowski, superdotação

\section{Contributions of the Theory of Positive Disintegration to the Field of Giftedness}

\begin{abstract}
Dabrowski`s Theory of Positive Disintegration (TPD) is taken as an important tool to understand the emotional development of gifted children. It is a theory of personality development which emphasizes the role of emotions in the human developmental potential. The purpose of this study is to present the central concepts of TPD and their contributions to the field of giftedness. It is emphasized the concept of overexcitabilites, which, besides being regarded as an indicator of giftedness, represents a key construct to the development of measures and to the promotion of interventions which focus is the personality and emotional characteristics of gifted children.
\end{abstract}

Keywords: Theory of Positive Disintegration, Dabrowski, giftedness

A Teoria da Desintegração Positiva (TDP), proposta por Kazimierz Dabrowski, é uma teoria da personalidade que apresenta contribuições significativas para a compreensão do desenvolvimento emocional de indivíduos superdotados. É preciso destacar que dois dos construtos enfatizados pela TDP, ou seja, personalidade e emoções, têm sido bem menos investigados que variáveis intelectivas, como a inteligência, por exemplo, quando se trata das pesquisas empíricas sobre superdotação. Uma simples consulta à base de dados PsycINFO (American Psychological Association [APA], 2013) - uma das mais relevantes da área de Psicologia - corrobora a afirmação anterior (Figura 1). Há cinco vezes mais investigações empíricas sobre a inteligência dos superdotados (195) do que sobre suas emoções e personalidade (39), embora, no geral, existam três vezes menos estudos empíricos sobre a primeira variável (6.838) quando comparados à soma das outras duas (22.566).

A TDP, segundo Jackson, Moyle e Piechowski (2009) e Silverman (2008), foi introduzida no campo da superdotação em 1979, em dois capítulos do livro New Voices in Counseling the Gifted, organizado por Colangelo e Zaffran, e tem recebido uma atenção crescente por parte dos estudiosos dos aspectos emocionais da superdotação, como atestam as

1 Endereço para correspondência: Universidade Federal de Juiz de Fora, Instituto de Ciências Humanas, Campus Universitário São Pedro, Rua José Lourenço Kelmer, s/n, Juiz de Fora, MG, Brasil. CEP. 36.001-970. E-mail: julianaoliveirapsi@gmail.com.

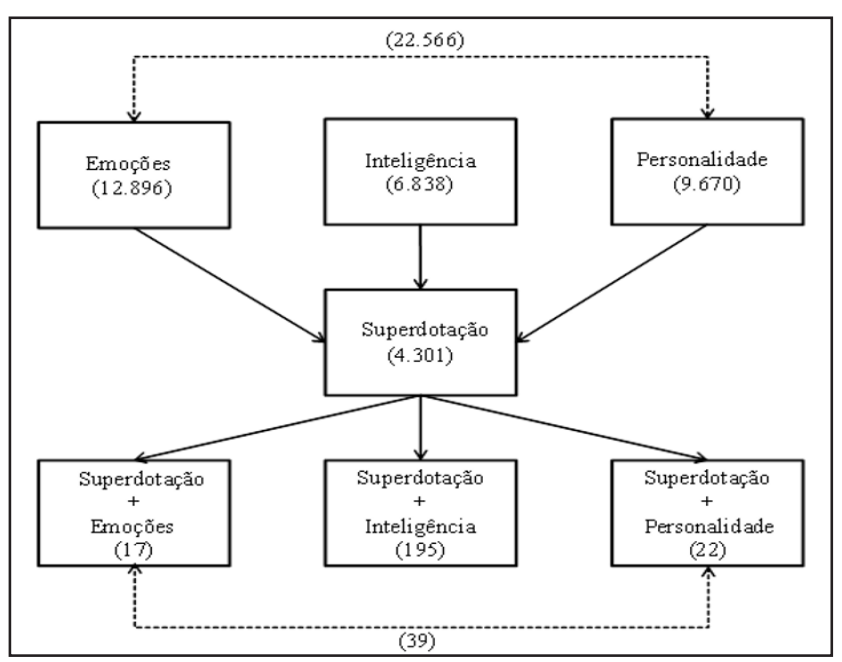

Figura 1. Estudos empíricos indexados pela PsycINFO

Nota. foram usados, evidentemente, os termos em inglês

numerosas publicações e trabalhos sobre ela apresentados em congressos da área. Ressalta-se que aproximadamente 13\% $(\mathrm{n}=5)$ das pesquisas empíricas indexadas pela PsycINFO (APA, 2013) sobre aspectos emocionais ou personalidade dos superdotados se baseiam nessa teoria. Não obstante, há 102 investigações empíricas indexadas nessa base que contêm termos chaves da TDP. Ainda assim, trata-se de uma perspectiva teórica pouco conhecida no Brasil. Desse modo, 
este artigo apresenta dados biográficos de Dabrowski e, em seguida, discorre a respeito desta teoria da personalidade e suas contribuições para a compreensão do desenvolvimento emocional de indivíduos superdotados.

\section{Dabrowski: Trajetória de Vida}

Kazimierz Dabrowski nasceu em Lublin, Polônia, em 1902. Passou os seus primeiros anos em um ambiente enriquecido, com expressiva exposição a livros e a música. Nos anos universitários, inicialmente em Lublin, estudou Psicologia, Filosofia e Literatura. Continuou os seus estudos em Genebra, com Édouard Claparède e Jean Piaget, tendo, na Universidade de Genebra, concluído o curso de Medicina e recebido o certificado de Pedagogia. Em 1930, estudou Psicanálise em Viena, tendo participado de reuniões com os psicanalistas mais conhecidos da época, entre eles, Freud. No ano seguinte, dedicou-se ao estudo da Psiquiatria Infantil em Paris. De 1933 a 1934, atendendo convite da Fundação Rockefeller, esteve na Universidade de Harvard, estudando saúde pública, voltando a seguir para a Suíça, onde por um ano foi professor de Psiquiatria Infantil. Em 1935, fundou o Instituto Polonês de Higiene Mental, em Varsóvia, tendo sido diretor desse instituto de 1935 a 1948, com um período interrompido durante a ocupação nazista. De origem judaica, passou vários meses preso em 1942, voltando novamente a ser prisioneiro por 18 meses, durante o regime comunista na Polônia em 1950 e 1951.

Dabrowski tinha grande pendor para a música, tendo inclusive, quando jovem, contemplado a possibilidade de ser um músico profissional. Escreveu também poemas, alguns deles traduzidos para o inglês e incluídos em texto sobre a sua criatividade (Amend, 2008).

No início dos anos de 1960, voltou aos Estados Unidos, tendo visitado a Universidade de Brandeis, onde trabalhava Abraham Maslow, um dos expoentes da Psicologia Humanista, com quem manteve intenso contato e discussões sobre as respectivas ideias teóricas. Em 1964, mudou-se para Montreal, onde trabalhou em um hospital e, no ano seguinte, tornou-se professor visitante da Universidade de Alberta, em Edmond, Canadá. Nesse mesmo ano, publicou o primeiro livro sobre a TDP: Positive Disintegration.

Foi considerado pela revista Psychology Today, em 1967, um dos psicólogos e psiquiatras mais importantes da história da ciência. Esse reconhecimento se deu, em grande parte, pela criação da TDP e por demonstrar os aspectos positivos das desordens mentais como mecanismos de desenvolvimento (Kobierzycki, 2010). Em 1979, após um ataque cardíaco, voltou para a Polônia, onde faleceu em 1980 (Tillier, 2008).

Após a morte de Dabrowski, um de seus principais colaboradores - Piechowski - deu continuidade aos estudos sobre a TDP e foi um dos responsáveis por essa teoria ser fortemente apropriada pela área de superdotação. Trata-se, segundo Piechowski (2008), da perspectiva teórica que mais tem ajudado a compreender o desenvolvimento emocional dos superdotados. Além desse autor, diversos pesquisadores - Frank Falk, Bill Tillier, Nanci Miller, Linda Silverman, Sal Mendaglio, Cheryl Ackerman, entre outros (p. ex., Kane, 2009) -, têm contribuído para corroborar a TDP.

\section{A Teoria da Desintegração Positiva}

Reitera-se que a publicação inaugural de Dabrowski sobre a TDP data de 1964, mas, já em um dos seus primeiros trabalhos (Dabrowski, 1937), é possível perceber as primeiras ideias dessa teoria, que evoluiu ao longo de muitos anos, a partir de diversas publicações (Dabrowski, 1967, 1972, 1973, 1996; Dabrowski, Kawczak, \& Piechowski, 1970) que a sustentaram e a complementaram. Atualmente, um amplo acervo da obra dabrowskiana, no idioma original e em língua inglesa, é disponibilizado em formato digital por Tillier (2013).

Um dos estudos de relevância para dar suporte empírico à TDP foi desenvolvido por Dabrowski de 1969 a 1972, com a colaboração especial de Piechowski. No referido estudo, redação de uma autobiografia, questões abertas a estímulos verbais, como incerteza, solidão, morte, ideal, sucesso, imortalidade, além de um teste de inteligência, exame neurológico e uma entrevista clínica foram utilizados para coleta de dados junto a voluntários. Com formação em neurologia, Dabrowski considerava o exame neurológico de fundamental importância, uma vez que, para ele, à medida que a pessoa alcança níveis mais altos de desenvolvimento, mudanças correspondentes ocorreriam no sistema nervoso. Uma descrição detalhada desse projeto, que contribuiu para estabelecer a estrutura dos níveis de desenvolvimento da personalidade, além da definição dos dinamismos e potencial de desenvolvimento, elementos chaves de sua teoria, é apresentada por Piechowski (2008).

Mendaglio (2008) afirma que a TDP tem sido considerada uma teoria da personalidade para o século XXI. Ela busca explicar o processo de desenvolvimento humano, atribuindo importância fundamental às emoções, uma vez que elas influenciam e direcionam o desenvolvimento da personalidade (Mendaglio, 2008, 2011), relevando a inteligência a uma posição secundária.

O desenvolvimento humano, na perspectiva da TDP, não é dividido em estágios fixos, organizados por faixas etárias, mas sim de acordo com um continuum hierárquico de níveis, denominados multiníveis, ou seja, em uma progressão de um baixo e primitivo nível para um alto e avançado nível. Dabrowski (1970b) descreveu cinco níveis de desenvolvimento da personalidade, que serão detalhados mais adiante. A transição entre níveis não é automática, ela tende a acontecer quando há movimento, quando ocorrem desintegrações e reestruturações.

Para a TDP, o indivíduo não necessita atender aos critérios de um nível específico para passar para o seguinte: pode-se estar em um nível de desenvolvimento em alguns aspectos e em outro nível no que diz respeito a outros aspectos (Frank, Gatto-Walden, \& Silverman, 2013; Mendaglio, 2008; Piechowski, 2008). Ackerman (2009) e Mendaglio (2008) ressaltam que, para a teoria, a idade não é um indicador do nível de desenvolvimento, podendo indivíduos mais jovens apresentar um desenvolvimento mais avançado. Esses autores destacam ainda que os níveis de desenvolvimento não são universais, sendo muito raros os indivíduos que alcançam o nível mais elevado. Porém, Dabrowski (1964) postulou que todo ser humano possui um instinto desenvolvimental, 
isto é, uma tendência a evoluir do menor ao maior nível de personalidade.

Na TDP o termo desintegração é utilizado porque a estrutura da personalidade existente tende a se desfazer. A desintegração é denominada positiva se contribui para o crescimento do indivíduo. Não obstante, desintegrações negativas - uma involução de nível - também podem ocorrer (Dabrowski, 1972, 1973; Figura 2).

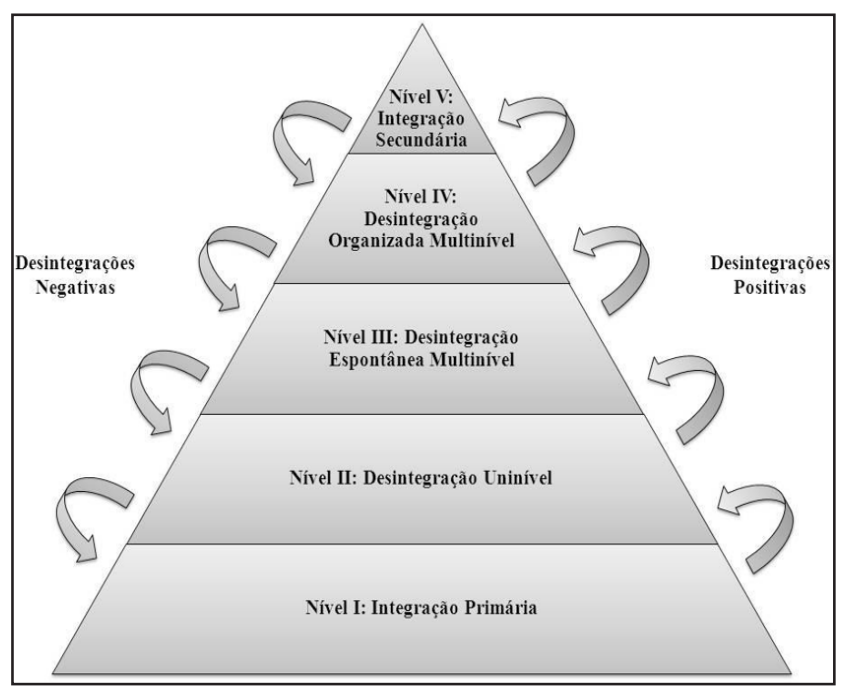

Figura 2. Desenvolvimento humano por meio de desintegrações positivas e negativas

Os cinco diferentes níveis de desenvolvimento da personalidade refletem diferenças nas características psicológicas dos indivíduos, que, por sua vez, são decorrentes do potencial de desenvolvimento (Mendaglio \& Tillier, 2006). Esse potencial é definido como a dotação inicial que determina o nível de desenvolvimento que uma pessoa pode atingir quando as condições físicas e ambientais forem ótimas (Dabrowski, 1996; Piechowski, 1975).

Três conjuntos de fatores controlam o desenvolvimento individual (Dabrowski, 1970a). O primeiro conjunto é determinado biologicamente e engloba os genes, as alterações permanentes na constituição física do organismo, as características psíquicas constitucionais e as potencialidades inatas do organismo. O segundo se refere às influências culturais e do ambiente social exercidas pelos indivíduos, isoladamente, ou por grupos, tais como família, amigos, instituições educacionais e religiosas, entre outras. $\mathrm{O}$ terceiro conjunto de fatores diz respeito aos processos autônomos que uma pessoa traz em seu desenvolvimento, tais como conflitos internos, autoconsciência e escolhas em relação ao crescimento pessoal. Os fatores autônomos são imprescindíveis para a formação de uma pessoa livre, independente e autêntica (Dabrowski, 1996).

O potencial de desenvolvimento não é composto, necessária e equitativamente, pelos três conjuntos de fatores (Dabrowski, 1996). Ele pode ser limitado ao primeiro ou aos dois primeiros conjuntos (Piechowski, 1974). Inclusive, para muitos indivíduos, o desenvolvimento é restrito somente às influências biológicas e sociais (Piechowski, 1975). Por conseguinte, nem todas as pessoas apresentam o terceiro conjunto no seu processo de desenvolvimento e as que apresentam não estão isentas das influências dos dois primeiros fatores (Dabrowski, 1996). No entanto, os processos autônomos representam uma nova capacidade do sujeito, pois podem fazer com que se aceitem alguns impulsos inatos (primeiro conjunto) e alguns padrões sociais (segundo conjunto) enquanto outros são rejeitados. Esses conjuntos de fatores podem ser observados de maneiras distintas nos diferentes níveis de desenvolvimento. Quando o terceiro conjunto passa a influenciar o desenvolvimento, o indivíduo começa a diferenciar entre as trajetórias inferiores e superiores de pensamento, emoção e ação, e uma autêntica hierarquia dos valores fica evidenciada (Dabrowski, 1970a). Portanto, os processos autônomos transcendem os ditames da biologia e da sociedade (Dabrowski, 1996).

Dabrowski $(1972,1973)$ também observou que o terceiro conjunto de fatores e alguns dinamismos são condições sine qua non para atingir níveis mais altos de desenvolvimento. Para o autor, dinamismos são forças biológicas ou de natureza mental que impulsionam o processo de desintegração positiva, auxiliando no controle do comportamento e na efetivação do potencial de desenvolvimento das pessoas. O autor (Dabrowski, 1972) descreveu-os como instintos, impulsos e processos intelectuais combinados às emoções. Alguns dinamismos referem-se às experiências de emoções negativas intensas de uma pessoa, tais como culpa e vergonha. Essas experiências contribuem para estimular mudanças de atitudes e percepções em relação a si e ao ambiente social.

A expressão das emoções, bem como dos comportamentos e pensamentos, também é qualitativamente diferente nos cinco níveis de desenvolvimento, sendo baseada em valores individuais ou grupais (Dabrowski, 1970a). Podem existir valores considerados extremamente elevados, como o altruísmo - característicos dos níveis mais altos de desenvolvimento -, e aqueles considerados muito inferiores, como, por exemplo, o egocentrismo -, próprios dos níveis mais baixos. Para Dabrowski (1970a), esses valores extremos podem ser melhor observados quando se consideram os multiníveis. Os cinco níveis são descritos a seguir de acordo com Dabrowski (1970b, 1996) e Piechowski (1975).

1. Nível I: Integração Primária - O primeiro nível se caracteriza por uma estrutura unitária e integrada. As pessoas que estão nele apresentam uma personalidade coesa, mas sem conflito interno e autorreflexão. São automáticas e impulsivas, determinadas por impulsos primitivos e inatos, apresentando o autointeresse como principal motivação. Pode haver indivíduos com algum grau de altruísmo; no entanto, ele não é genuíno, seus comportamentos são orientados para a satisfação das necessidades básicas e são, em todos os aspectos, egocêntricos.

2. Nível II: Desintegração Uninível - A desintegração uninível inicia-se com o afrouxamento da rígida estrutura de integração primária. A partir desse nível, começa-se a exibir graus de desintegrações parciais e desintegrações de estruturas psicológicas. A rigidez, existente no primeiro patamar, vai sendo substituída por hesitação, dúvida, atitudes vacilantes e mudanças. Os indivíduos que estão nesse nível são sensíveis à opinião social. Sentimentos de vergonha 
e culpa começam a ser experimentados em relação às expectativas externas. No entanto, esses sentimentos não impulsionam as pessoas para a reflexão interna e para uma hierarquização de valores.

3. Nível III: Desintegração Espontânea Multinível Este é o primeiro nível em que uma hierarquia de valores começa a se formar e o terceiro fator (fatores autônomos) passa a atuar no desenvolvimento, permitindo uma autodeterminação. Pode-se notar a formação de uma consciência crítica de si mesmo e das outras pessoas, além de uma insatisfação do indivíduo entre "o que se é" e "o que se deveria ser”. Sentimentos de autoavaliação e autorreflexão, conflitos morais intensos, percepção da singularidade dos outros e ansiedade existencial são característicos desse nível de desenvolvimento.

4. Nível IV: Desintegração Organizada Multinível Durante o desenvolvimento do nível IV, o processo de crescimento torna-se mais consciente e o indivíduo tem mais responsabilidade pelo seu crescimento. Há uma elevada diferenciação dos fatores autônomos; o conflito interno diminui à medida que a pessoa se torna mais autêntica e a diferença entre "o que é" e "o que deveria ser" passa a ser mais estreita, ao buscar um "ideal de personalidade". Nesse nível, há um crescimento acentuado de sentimentos de empatia, autoconhecimento e autocontrole.

5. Nível V: Integração Secundária - $\mathrm{O}$ último nível se caracteriza por uma integração secundária. Essa última etapa consiste em uma nova organização e harmonização da personalidade. As funções cognitivas e emocionais são fundidas em uma união harmoniosa e flexível. Há carência de conflito interno, uma vez que não há mais uma diferença entre "o que é" e "o que deveria ser". Esse nível é marcado pela autonomia, autenticidade e empatia altamente refinada. As pessoas que estão nele podem ser caracterizadas por uma compaixão universal e pelo autossacrifício.

De acordo com Dabrowski (1972), o potencial de desenvolvimento pode ser acessado com base nos seguintes componentes: (a) habilidades e talentos especiais que os indivíduos podem manifestar; (b) fatores autônomos (terceiro fator); e (c) sobre-excitabilidade (SE) psíquica. No entanto, a maior evidência e a principal manifestação do potencial de desenvolvimento dizem respeito aos cinco tipos de SE (Dabrowski, 1972).

\section{Sobre-excitabilidade}

O termo SE ou SE psíquica foi utilizado pela primeira vez por Dabrowski em 1937 (Dabrowski, 1937), sendo posteriormente (Dabrowski, 1972) conceituado como tendências de os indivíduos reagirem com extrema intensidade e sensibilidade a diversos estímulos, sejam eles externos ou internos. Essa forma ampliada e intensificada de reagir pode ocorrer em uma ou mais das seguintes áreas ou padrões: Sensorial, Psicomotora, Intelectual, Imaginativa e Emocional (Dabrowski, 1972). Os cinco padrões de SE são descritos brevemente a seguir, com base nos estudos de Dabrowski (1972; 1996), Piechowski (1975) e Falk, Lind, Miller, Piechowski e Silverman, (1999):

1. SE Psicomotora: Esse tipo de SE pode se manifestar em duas formas: em função de um excesso de energia do organismo ou pela excessiva excitabilidade do sistema neuromuscular. Indivíduos com esse padrão de sobre-excitabilidade caracterizam-se por alto grau de energia física, são ativos, agitados, impulsivos, muito falantes e com dificuldade em se manter parados. O excesso de energia exibido pode resultar em habilidades atléticas, mas não necessariamente deve ser confundido com elas.

2. SE Sensorial: Diz respeito à elevada diferenciação e vivacidade de experiências sensoriais. É expressa por indivíduos que apresentam reações intensas derivadas de um ou mais sentidos (tato, olfato etc.). Sensibilidade e elevado interesse em componentes estéticos de objetos, tais como os observados em suas cores, formas e texturas ou outros prazeres sensoriais, tais como aromas e sabores, também são experimentados

3. SE Imaginativa: É expressa no gosto pelo inusitado e pela facilidade para fantasiar e/ou sonhar. Indivíduos com SE Imaginativa apresentam pensamentos criativos e têm maior facilidade para realizar criações poéticas, inventar histórias etc. Além disso, manifestam ricas associações de imagens e impressões, utilizam metáforas e outras figuras de linguagem na expressão verbal, conseguindo experimentar a realidade de modos não convencionais.

4. SE Intelectual: É manifestada pelo interesse por conhecimento e pela persistência em buscar respostas para soluções de diversos problemas. Indivíduos com SE Intelectual se caracterizam pela curiosidade, pensamento analítico, independência de pensamento, observação aguçada, facilidade em formular novos conceitos e respostas lógicas, predisposição a realizar análises teóricas e reflexões filosóficas, entre outros.

5. SE Emocional: Evidencia-se em relações afetivas caracterizadas por um forte apego a pessoas e outros seres vivos. Os indivíduos que manifestam esse padrão de SE apresentam elevada intensidade emocional e uma gama diferenciada de sentimentos, afetos e paixões, além de demonstrarem alto senso de responsabilidade social e empatia.

Dabrowski (1972) apresentou os padrões de SE de forma hierarquizada, ou seja, alguns (Emocional, Imaginativo e Intelectual) são mais elevados que outros (Sensorial e Psicomotor) em relação ao processo de desenvolvimento humano. A SE Emocional é de fundamental importância na formação de uma hierarquia de valores, empatia, autoconsciência, entre outros dinamismos que desempenham um papel decisivo no desenvolvimento humano; a SE Imaginativa tem grande importância na criatividade artística, no planejamento intuitivo etc.; e a SE Intelectual dá origem à criatividade acadêmica e possibilita reflexão, autocontrole, autonomia e autenticidade (Dabrowski, 1973). Juntas, essas três formas dão ricas possibilidades de crescimento e servem 
para transformar os outros padrões de SE (Dabrowski, 1972). $\mathrm{O}$ autor acrescenta que, se combinadas às SEs Sensorial e Psicomotora, os três padrões mais elevados fazem com que o indivíduo enriqueça ainda mais e aprimore as possibilidades de um desenvolvimento positivo. Desse modo, o potencial desenvolvimental será mais forte se todas - ou quase todas - as formas de SE estiverem presentes (Dabrowski, 1996).

\section{Teoria da Desintegração Positiva e Superdotação}

Apesar de a TDP não ser uma teoria específica sobre superdotação, ela tem influenciado várias pesquisas sobre esse construto (Mendaglio, 2008) e tem fornecido uma excelente estrutura para a caracterização dos superdotados e para a compreensão de seu desenvolvimento emocional (Ackerman, 2009; Silverman, 2008). Pyrit e Mendaglio (2007) ressaltam que a TDP representa uma base conceitual para os interessados no desenvolvimento holístico de pessoas superdotadas. Mais do que habilidade intelectual e desempenho, aspectos mais focalizados na educação do superdotado, emoção, empatia, autoconsciência, consciência do outro e comportamento altruístico são enfatizados.

Reitera-se que grande parte dos pressupostos da TPD foi desenvolvido a partir das experiências de Dabrowski em atendimentos clínicos e estudos biográficos de artistas, escritores, membros de ordens religiosas e indivíduos com superdotação (Kobierzycki, 2000; Tillier, 2008). Em suas pesquisas, Dabrowski (1967) propôs a hipótese de que alguns indivíduos, especialmente as pessoas criativas e com talentos, tendem a possuir níveis mais altos de potencial de desenvolvimento. Para o autor, um indivíduo com superdotação ou submetido a um processo de aceleração de desenvolvimento é mais propenso a exibir processos desintegrativos, podendo se tornar parte de um processo geral de desintegração positiva - concluir um nível específico - ou, até mesmo, culminar em uma integração secundária - alcançar o quinto nível (Dabrowski, 1970b). Em muitos casos, esse potencial predispõe a pessoa para um crescimento acelerado, contribuindo para o desenvolvimento de uma personalidade eminente (Dabrowski, 1972).

No entanto, Mendaglio (2002) sinaliza que os indivíduos com elevado potencial de desenvolvimento nem sempre satisfazem os critérios para serem identificados como superdotados por programas tradicionais. Além disso, nem todas as pessoas consideradas possuidoras de superdotação por esses programas têm um alto potencial desenvolvimental (Mendaglio, 2002).

Tillier (2002) analisa a superdotação considerando os conjuntos de fatores que controlam o desenvolvimento humano. Para o autor, o primeiro conjunto canaliza a superdotação para o cumprimento de metas pessoais; muitas vezes, a custo dos outros e de forma antissocial. O segundo molda a criatividade para que ela siga e apoie o meio social (Tillier, 2002). Portanto, restringe a criatividade, desencorajando sua expressão e o pensamento individual.

Nesse sentido, pessoas com níveis mais baixos de desenvolvimento usam seus talentos para apoiar objetivos egocêntricos; suas habilidades e talentos especiais tendem a atuar no nível de desenvolvimento individual. À medida que estão em níveis mais elevados de desenvolvimento, esses indivíduos canalizam seus talentos e habilidades para sua hierarquia de valores pessoais, expressando sua visão de personalidade ideal e de como o mundo deveria ser (Tillier, 2002).

O terceiro conjunto de fatores adquire importância fundamental no potencial de desenvolvimento. Ele direciona o talento e a criatividade para expressões autônomas e fornece motivação para o indivíduo lutar por metas que, muitas vezes, podem estar além de seu próprio alcance (Tillier, 2002). Com a influência desse fator, a superdotação pode ser aplicada a serviço de altos valores, como compaixão, solidariedade, altruísmo etc., de modo mais consciente e crítico. Piechowski (1974) também salientou que indivíduos com muitos talentos apresentam os componentes autônomos (terceiro conjunto de fatores) particularmente fortes, contribuindo, assim, para maior potencial de desenvolvimento.

\section{Sobre-excitabilidade e Superdotação}

Dos diversos conceitos atrelados à TDP, o de SE é o que mais tem beneficiado o campo de estudos de superdotação (Ackerman \& Nian, 2008). Em um estudo de 1962, Dabrowski chegou à conclusão de que indivíduos criativos e com superdotação tendem a possuir níveis mais altos de SE (Dabrowski, 1972). Além disso, eles se diferenciavam pela maneira de aliviar a tensão e responder à estimulação (Silverman, 2008). Reitera-se que o autor fez diversas observações, entrevistas, estudos de caso e realizou uma série de exames e testes psicológicos, psiquiátricos e neurológicos em crianças e jovens previamente identificados como superdotados nas áreas intelectual ou artística.

Para Piechowski (1979), as SEs são componentes básicos da superdotação, compartilhadas por muitos superdotados e têm sido, desde então, incluídas na descrição desses indivíduos por numerosos autores, como Tannenbaum (1983, 1990), Davis e Rimm (1994), Coleman e Cross (2000) e Renzulli (2003). No entanto, o autor destaca que as SEs não podem ser consideradas domínios específicos de talento; ao invés disso, elas representam um componente da superdotação que nutre, fortalece e amplifica o desempenho das pessoas que possuem essa característica (Piechowski \& Colangelo, 1984).

Desde as proposições de Dabrowski e Piechowski, vários estudos foram conduzidos para investigar as SE em indivíduos com superdotação (Gross, Rinn, \& Jamieson, 2007; Miller, Falk, \& Huang, 2009; Moon \& Montgomery, 2005; Rinn, Mendaglio, Rudasill, \& McQueen, 2010; Rinn \& Reynolds; 2012; Piechowski \& Miller, 1995; Tieso, 2007b; Treat, 2006; entre outros) ou para comparar os escores de pessoas com e sem essa condição (Ackerman, 1997; Al-Onizat, 2013; Bouchard, 2004; Bouchet \& Falk, 2001; Carman, 2011; Gallagher, 1986; Harrisson \& Haneghan, 2011; Miller et al., 2009; Piechowski, Silverman, \& Falk, 1985; Piirto \& Frass, 2012; Sanz, 2006; Siu, 2010; Tieso, 2007a; Wirthwein \& Rost, 2011; Yakmaci-Guzel \& Akarsu, 2006). Essas investigações têm demonstrado que os superdotados tendem a apresentar níveis mais altos de SE em um ou mais padrões. Há pesquisas, inclusive, que identificaram níveis elevados de 
todos os padrões de SE em pessoas superdotadas (Carman, 2011; Miller, Silverman, \& Falk, 1994; Piechowski et al., 1985; Siu, 2010) e que evidenciaram associação entre SE e volume da massa cinzenta de certas áreas do cérebro de estudantes talentosos (Chang \& Kuo, 2013).

Para mensurar as cinco formas de SEs, diversas medidas têm sido empregadas tais como o Overexcitability Questionnaire (Lisy \& Piechowski, 1983; Piechowski, 1996), o ElemenOE (Bouchard, 2004), a Me Scale (Chang \& Kuo, 2009) e o Overexcitability Questionnaire Two (OEQ-II; Falk et al., 1999). Destaca-se que a última é, atualmente, a medida de SE mais utilizada para identificar superdotação (Bouchet \& Falk, 2001; Carman, 2011; Siu, 2010; Tieso, 2007a; Wirthwein \& Rost, 2011), além de ser a que apresenta mais estudos sobre suas evidências de validade e fidedignidade (Carman, 2011; Gross et al., 2007; Tieso, 2007a, 2007b; Wirthwein \& Rost, 2011). Há, pelo menos, sete países que possuem versões traduzidas e/ou adaptadas do OEQ-II: Espanha (Sanz, 2006), Coréia do Sul (Piirto, Montgomery, \& May, 2008), Turquia, México (Falk, Yakmaci-Guzel, Chang, Sanz, \& Chavez-Eakle, 2008), Hong Kong (Siu, 2010), Jordânia (Al-Onizat, 2013) e Brasil (Oliveira, 2013).

É preciso considerar, porém, que nem todas as formas de SE possibilitam maior desenvolvimento ou trazem benefícios aos sujeitos (Mendaglio, 2012a, 2012b). Isso não é diferente quando se refere a indivíduos com superdotação. Embora, de modo geral, elas possam contribuir para um desenvolvimento individual acelerado, em algumas pessoas, as SEs podem criar tensões e levar a sérios problemas psicológicos (Mika, 2005). Tillier (2002) relembra que Dabrowski se referiu às SEs como "um dote trágico" para assinalar que a "estrada" de uma pessoa com forte SE não é necessariamente fácil ou suave.

Deve-se, portanto, atentar-se quanto a alguns aspectos do desenvolvimento das pessoas que exibem elevada SE, especialmente pessoas com superdotação, pois, em níveis elevados, as intensidades e sensibilidades podem aparecer em desacordo com os pares de mesma idade, levando-os a apresentarem uma série de questionamentos e conflitos a respeito de si (O'Connor, 2002). Aqueles que apresentam elevada SE Psicomotora, por exemplo, podem manifestar, aparentemente, uma variedade de comportamentos semelhantes à hiperatividade e, em muitos casos, pode até ser confundida com ela (Mendaglio, 2002; Mika, 2006). Pessoas que buscam constantemente prazeres sensoriais podem exibir exagerada busca por conforto, beleza, luxúria etc., o que não contribui para alcançar maiores níveis de desenvolvimento (Piechowski \& Colangelo, 1984). Já alunos com SE Intelectual muito elevada podem apresentar questionamentos incessantes para satisfazer sua curiosidade, representando, assim, desafios para educadores (Bailey, 2010; Mendaglio, 2002). Pessoas com forte e desequilibrada SE Imaginativa estão mais propensas a ilusões e sonhos, transes hipnóticos e, até mesmo, alucinações (Mika, 2005). Por fim, indivíduos com alta SE Emocional são mais predispostos a ter emoções intensas, devido à grande preocupação com os outros e consciência dos acontecimentos globais (Mendaglio, 2002).

Tieso (2007b) salienta a necessidade de examinar as SEs e promover estratégias de intervenção que reforcem as características positivas das pessoas, auxiliando-as a compensar os efeitos negativos que podem ser acarretados por elas. Essas intervenções podem ser realizadas precocemente, pois, de acordo com Dabrowski (1972), quase todas as formas podem ser percebidas desde cedo, na infância. Em crianças de um e dois anos, já é possível observar alguns padrões e, quanto maior a idade, mais fácil fica distingui-los.

Desse modo, o aconselhamento psicológico, a psicoterapia e o contexto educacional representam campos nos quais a TDP e, especialmente, as SEs devem ser trabalhadas (Bailey, 2010; Ogburn-Colangelo, 1989; Strickland, 2000). Uma descrição do uso dessa teoria direcionando o processo psicoterapêutico e de aconselhamento é apresentada por Jackson, Moyle e Piechowski (2009) e Jackson e Moyle (2009). Ao aceitar e compreender o significado de seus sentimentos, crises e processos desenvolvimentais, os indivíduos com superdotação podem seguir em busca de seu próprio desenvolvimento (Tillier, 2002).

\section{Considerações Finais}

Há que se alertar que a TDP não se restringe à SE e nem ao estudo da superdotação. Evidentemente, como todos os sistemas teóricos, essa teoria do desenvolvimento da personalidade possui limitações. Salienta-se, por um lado, que ela não é capaz de explicar a personalidade e todas as caraterísticas emocionais de todos os superdotados. Por outro lado, é possível perceber algumas circunscrições referentes às evidências empíricas necessárias para corroborá-la. Entre outras, destacam-se aquelas relacionadas à etiologia da SE e à sua manifestação ao longo do curso de vida. Apesar de, como apresentado, terem sido identificadas estruturas cerebrais associadas à SE, não se sabe ainda se elas antecedem ou constituem uma consequência desse modo de perceber e reagir intensamente perante estímulos. Além disso, ao considerar, de acordo com Dabrowski (1964), que a SE é inata, há que se conduzirem investigações para mapear seus marcadores genéticos. Ainda que sejam encontrados alguns estudos longitudinais (Peterson, 2012), pouco se sabe sobre a estabilidade e a mudança dos padrões de SE durante a vida, especialmente na velhice. Detectou-se na PsycINFO somente uma investigação (Falk, Manzanero, \& Miller, 1997) sobre SE que incluiu um idoso na amostra.

Não obstante as limitações, é possível afirmar que se trata de um referencial que lança luz sobre os aspectos emocionais de pessoas com superdotação (Ogburn-Colangelo, 1989; Piechowski, 1997; Schiever, 1985). Constitui, ademais, uma base sólida para o aconselhamento e a psicoterapia destinados aos superdotados (Mendaglio, 2008), bem como para o desenvolvimento de medidas para identificá-los (Falk \& Miller, 2008; Mendaglio \& Tillier, 2006).

Para finalizar, destacamos, a seguir, as palavras de Daniels (2009) a respeito das contribuições da TDP para o campo da educação do superdotado:

O trabalho de Dabrowski oferece uma base teórica para: (1) reconhecer aspectos do desenvolvimento da personalidade no superdotado; (2) passar a perceber características que frequentemente são vistas como inadequadas e problemáticas em uma perspectiva mais positiva - como aspectos essenciais do potencial de desenvolvimento do indivíduo; (3) conduzir 
novos estudos relacionados ao desenvolvimento emocional e da personalidade de crianças superdotadas. Ademais, a TDP é de fundamental importância para uma nova perspectiva sobre o desenvolvimento ao longo da vida de individuos superdotados. (p. 262)

\section{Referências}

Ackerman, C. M. (1997). Identifying gifted adolescents using personality characteristics: Dabrowski's overexcitabilities. Roeper Review, 19, 229-236.

Ackerman, C. M. (2009). The essential elements of Dabrowski's theory of positive disintegration and how they are connected. Roeper Review, 31, 81-95.

Ackerman, C. M., \& Nian, Q. (2008). What interdisciplinary literature on Dabrowski's theory can offer gifted education. Poster session presented at the 55th Annual Convention of the National Association for Gifted Children, Tampa, FL. Disponível em http://khup.com/download/3_keyword-interdisciplinarystudies-theory-offered/what-interdisciplinary-literature-ondabrowski-039-s-theory-can.pdfAcesso em 15 de abril de 2013.

Al-Onizat, S. H. (2013). The psychometric properties of a Jordanian version of Overexcitability Questionnaire-Two, OEQII. Creative Education, 4, 49-61.

Amend, D. (2008). Creativity in Dabrowski and the theory of positive disintegration. In S. Mendaglio (Ed.), Dabrowski s theory of positive disintegration (pp. 123-137). Scottsdale, AZ: Great Potential Press.

American Psychological Association. (2013). APA PsycNET. Disponível em http://www.apa.org/pubs/databases/psycinfo/ index.aspx. Acesso em 7 de novembro de 2013.

Bailey, C. L. (2010). Overexcitabilities and sensitivities: Implications of Dabrowski's Theory of Positive Disintegration for counseling the gifted. Disponível em http://counselingoutfitters.com/ vistas/vistas10/Article_10.pdf. Acesso em 15 de abril de 2013.

Bouchard, L. L. (2004). An instrument for the measure of dabrowskian overexcitabilities to identify gifted elementary students. Gifted Child Quarterly, 48, 339-350.

Bouchet, N., \& Falk, R. F. (2001). The relationship among giftedness, gender, and overexcitability. Gifted Child Quarterly, 45, 260-267.

Carman, C. A. (2011). Adding personality to gifted identification: Relationships among traditional and personality-based constructs. Journal of Advanced Academics, 22, 412-446.

Chang, H. J., \& Kuo, C. C. (2009). Overexcitabilities of gifted and talented students and its related researches in Taiwan. AsiaPacific Journal of Gifted and Talented Education, 1, 41-74.

Chang, H., \& Kuo, C. (2013). Overexcitabilities: Empirical studies and application. Learning and Individual Differences, 23, 53-63.

Coleman, L. J., \& Cross, T. L. (2000). Social-emotional development and the personal experience of giftedness. In K. A. Heller, F. J. Mönks, R. J. Sternberg, \& R. F. Subotnik (Eds.), International handbook of giftedness and talent (pp. 203-212). Oxford: Elsevier.

Dabrowski, K. (1937). Psychological basis of self-mutilation. Genetic Psychology Monographs, 19, 1-104.

Dabrowski, K. (1964). Positive disintegration. Boston: Little Brown.
Dabrowski, K. (1967). Personality shaping through positive disintegration. London: Gryf.

Dabrowski, K. (1970a). Outline of the theory of positive disintegration. In K. Dabrowski, A. Kawczak, \& M. M. Piechowski (Eds.), Mental growth through positive disintegration (pp. 1726). London: Gryf.

Dabrowski, K. (1970b). Positive and accelerated development. In K. Dabrowski, A. Kawczak, \& M. M. Piechowski (Eds.), Mental growth through positive disintegration (pp. 27-61). London: Gryf.

Dabrowski, K. (1972). Psychoneurosis is not an illness: Neuroses and psychoneuroses from the perspective of positive disintegration. London: Gryf.

Dabrowski, K. (1973). The dynamics of concepts. London: Gryf Publications.

Dabrowski, K. (1996). Multilevelness of emotional and instinctive functions. Lublin, Poland: Towarzystwo Naukowe Katolickiego Uniwersytetu Lubelskiego.

Dabrowski, K., Kawczak, A., \& Piechowski, M. (1970). Mental growth through positive disintegration. London: Gryf.

Daniels, S. (2009). Under construction: Continued applications of Dabrowski`s theory of positive disintegration with the gifted. In S. Daniels \& M. M. Piechowski (Eds.), Living with intensity (pp. 261-265). Scottscale, AZ: Great Potential.

Davis, G. A., \& Rimm, S. B. (1994). Education of the gifted and talented (third edition). Needham Heights, MA: Allyn and Bacon.

Falk, R. F., Lind, S., Miller, N. B., Piechowski, M. M., \& Silverman, L. K. (1999). The Overexcitability Questionnaire Two (OEQ II). Manual, scoring system, and questionnaire. Denver: Institute for the Study of Advanced Development.

Falk, R. F., Manzanero, J. B., \& Miller, N. B. (1997). Developmental potencial in Venezuelan and American artists: A cross-cultural validity study. Creativity Research Journal, $10(2 \& 3), 201$ 206.

Falk, R. F., \& Miller, N. B. (2008). Building firm foundations research and assessments. In S. Daniels \& M. Piechowski (Eds.), Living with intensity (pp. 239-259). Scottsdale AZ: Great Potential Press.

Falk, R. F., Yakmaci-Guzel, B., Chang, A. H., Sanz, R. P. S., \& Chavez-Eakle, R. A. (2008). Measuring overexcitabily: Replication across five countries. In S. Mendaglio (Ed.), Dabrowski's theory of positive disintegragion (pp. 183-199). Scottsdale AZ: Great Potential Press.

Frank, J., Gatto-Walden, P., \& Silverman, L. (2013). Dabrowski `s theory of positive disintegration: A process of development. Comunicação apresentada na 20th Biennial World Conference, Louisville, Kentucky, agosto de 2013.

Gallagher, S. A. (1986). A comparison of the concept of overexcitabilities with measures of creativity and school achievement in sixth-grade students. Roper Review, 8, 115-119.

Gross, C. M., Rinn, A. N., \& Jamieson, K. M. (2007). Gifted adolescents' overexcitabilities and self-concepts: An analysis of gender and grade level. Roeper Review, 29, 240-248.

Harrison, G. E., \& Haneghan, J. P. V. (2011). The gifted and the shadow of the night: Dabrowski's overexcitabilities and their correlation to insomnia, death anxiety, and fear of the unknown. Journal for the Education of the Gifted, 34(4), 669-697. 
Jackson, P. S., \& Moyle, V. F. (2009). Integrating the intense experience: Counseling and clinical applications. In S. Daniels \& M. M. Piechowski (Eds.), Living with intensity (pp. 105125). Scottscale, AZ: Great Potential.

Jackson, P. S., Moyle, V. F., \& Piechowski, M. M. (2009). Emotional life and psychotherapy of the gifted in light of Dabrowski 's theory. In L. V. Shavinina (Ed.), International handbook on giftedness (pp. 437-466). Springer.

Kane, M. (2009). Contemporary voices on Dabrowski's theory of positive disintegration. Roeper Review, 31, 72-76.

Kobierzycki, T. (2000). Summaries: Professor Dr. Kazimierz Dabrowski (1902-1980). (A. Przybylek, Trans.). Heksis: Scientific-didactic quarterly devoted to problems of person, health, creativity and spirituality, 1-3 (22\&24), 276-279.

Kobierzycki, T. (2010). Biography of Kazimierz Dąrowski. Disponível em http://positivedisintegration.com/ Kobierzycki2010.pdf. Acesso em 15 de abril de 2013.

Lysy, K. Z., \& Piechowski, M. M. (1983). Personal growth: An empirical study using Jungian and Dabrowskian measures. Genetic Psychology Monographs, 108, 267-320.

Mendaglio, S. (2002). Dabrowski's theory of positive disintegration: Some implications for teachers of gifted students. AGATE, $15,14-22$.

Mendaglio, S. (2008). Dabrowski`s theory of positive disintegration: A personality theory for the 21 st century. In S. Mendaglio (Ed.), Dabrowski's theory of positive disintegration (pp. 13-40). Scottsdale, AZ: Great Potential Press.

Mendaglio, S. (2011). Emotions and giftedness. In T. L. Cross (Ed.), On the social and emotional lives of gifted children (pp. 58-61). Waco, Texas: Prufrock Press.

Mendaglio, S. (2012a). Overexcitabilities and giftedness research: A call for a paradigm shift. Journal for the Education of the Gifted, 35, 207-219.

Mendaglio, S. (2012b). Extreme sensitivity: Maladaptive strategy or defining characteristic. Comunicação apresentada na 59th Annual Convention, National Association for Gifted Children, Denver, Colorado, EUA, novembro de 2012.

Mendaglio, S., \& Tillier, W. (2006). Dabrowski's theory of positive disintegration and giftedness: Overexcitability research findings. Journal for the Education of the Gifted, 30, 68-87.

Mika, E. (2005). Theory of positive disintegration as a model of personality development for exceptional individuals. In N. L. Hafenstein, B. Kutrumbos, \& J. Delisle (Eds.), Perspectives in gifted education: Complexities of emotional development, spirituality and hope (pp. 33-59). Denver: University of Denver, Institute for the Development of Gifted Education, Ricks Center for Gifted Children.

Mika, E. (2006). Giftedness, ADHD, and Overexcitabilities: The possibilities of misinformation. Roeper Review, 28, 237-242.

Miller, N. B., Falk, R. F., \& Huang, Y. (2009). Gender identity and the overexcitability profiles of gifted college students. Roeper Review, 31, 161-169.

Miller, N. B., Silverman, L. K., \& Falk, R. E (1994). Emotional development, intellectual ability, and gender. Journal for the Education of the Gifted, 18, 20-38.

Moon, J., \& Montgomery, D. (2005). Profiles of overexcitabilities for Korean high school gifted students according to gender and domain of study. Journal of Gifted /Talented Education, 15, 1-10.
O'Connor, K. J. (2002). The application of Dabrowski's theory to the gifted. In M. Neihart, S. M. Reis, N. M. Robinson, \& S. M. Moon (Eds.), The social and emotional development of gifted children: What do we know? (pp. 51-60). Waco, TX: Prufrock Press.

Ogburn-Colangelo, M. K. (1989). Giftedness as multilevel potential: A clinical example. Advance Development Journal, $1,87-101$.

Oliveira, J. C. (2013). Sobre-excitabilidade e talento: Evidências de validade da versão brasileira do Overexcitability Questionnaire Two (Dissertação de mestrado), Programa de Pós-Graduação em Psicologia, Universidade Federal de Juiz de Fora, Juiz de Fora, MG.

Peterson, J. S. (2012). The asset-burden paradox of giftedness: A 15-year phenomenological, longitudinal case study. Roeper Review, 34, 244-260.

Piechowski, M. M. (1974). Two developmental concepts: Multilevelness and developmental potential. Counseling and Values, 18, 8693.

Piechowski, M. M. (1975). A theoretical and empirical approach to the study of development. Genetic Psychology Monographs, 92, 231-297.

Piechowski, M. M. (1979). Developmental potential. In N. Colangelo \& R. Zaffrann (Eds.), New voices in counseling the gifted (pp. 25-57). Dubuque, Iowa: Kendall Hunt.

Piechowski, M. M. (1996). Overexcitability Questionnaire Revised (OEQ-REV). In S. Mendaglio (Ed.), Dabrowski's theory of positive disintegration (pp. 269-270). Scottsdale, AZ: Great Potential Press.

Piechowski, M. M. (1997). Emotional giftedness: The measure of intrapersonal intelligence. In N. Colangelo \& G.A. Davis (Eds.), Handbook of Gifted Education (pp. 366-381). Boston: Allyn and Bacon.

Piechowski, M. M. (2008). Discovering Dabrowski`s theory. In S. Mendaglio (Ed.), Dabrowski s theory of positive disintegration (pp. 41-77). Scottsdale, AZ: Great Potential Press.

Piechowski, M. M., \& Colangelo, N. (1984). Developmental potential of the gifted. Gifted Child Quarterly, 28, 80-88.

Piechowski, M. M., \& Miller, N. B. (1995). Assessing developmental potential in gifted children: A comparison of methods. Roeper Review, 17, 176-180.

Piechowski, M. M., Silverman, L. K., \& Falk, F. (1985). Comparison of intellectually and artistically gifted on five dimensions of mental functioning. Perceptual and Motor Skills, 60, 539-549.

Piirto, J., \& Fraas, J. (2012). A mixed-methods comparison of vocational and identified-gifted high school students on the overexcitability questionnaire. Journal for the Education of the Gifted, 35, 3-34.

Piirto, J., Montgomery, D., \& May, J. (2008). A comparison of Dabrowski's overexcitabilities by gender for American and Korean high school gifted students. High Ability Studies, 19, 141-153.

Pyrit, M. C., \& Mendaglio, S. (2007). Dabrowski's theory of positive disintegration: What educators should know. Comunicação apresentada na 54th Annual Convention, National Association for Gifted Children, Minneapolis, EUA, novembro de 2007. 
Renzulli, J. S. (2003). Conception of giftedness and its relationship to the development of social capital. In N. Colangelo \& G. A. Davis (Eds.), Handbook of gifted education (pp. 75-87). Boston: Allyn and Bacon.

Rinn, A. N., Mendaglio, S., Rudasill, K. M., \& McQueen, K. S. (2010). Examining the relationship between the overexcitabilities and self-concepts of gifted adolescents via muitivariet cluster analysis. Gifted Child Quarterly, 54, 3-17.

Rinn, A. N., \&, Reynolds, M. J. (2012). Overexcitabilities and ADHD in the gifted: An examination. Roeper Review, 34, 38-45.

Sanz, R. P. S. (2006). El alumno superdotado y sus problemas de aprendizaje: validación del OEQ-II como prueba de diagnostic (Tese de Doutorado), Facultad de Educación, Universidad Complutense de Madrid, Madrid, Espanha. Disponível em http://eprints.ucm.es/7254/1/T26463.pdf. Acesso em 15 de abril de 2013.

Schiever, S. (1985). Creative personality characteristics and dimensions of mental functioning in gifted adolescents. Roeper Review, 7, 223-226.

Silverman, L. K. (2008). The theory of positive disintegration in the field of gifted education. In S. Mendaglio (Ed.), Dabrowski 's theory of positive disintegration (pp. 157-173). Scottsdale, AZ: Great Potential Press.

Siu, A. F. Y. (2010). Comparing overexcitabilities of gifted and non-gifted school children in Hong Kong: Does culture make a difference? Asia Pacific Journal of Education, 30, 71-83.

Strickland, C. (2000). Living and learning with Dabrowski's overexcitabilities. Disponível em: http://www.hoagiesgifted. org/strickland_dabrowski.pdf. Acesso em 15 de abril de 2013.
Tannenbaum, A. J. (1983). Gifted children. Psychological and educational perspectives. New York: Macmillan.

Tannenbaum, A. J. (1990). Giftedness: A psychosocial approach. In R. J. Sternberg \& J. E. Davidson (Eds.), Conceptions of giftedness (pp. 21-52). Cambridge: Cambridge University Press.

Tieso, C. L. (2007a). Overexcitabilities: A new way to think about talent? Roeper Review, 29, 232-239.

Tieso, C. L. (2007b). Patterns of overexcitabilities in identified gifted students and their parents: A hierarchical model. Gifted Child Quarterly, 51, 11-22.

Tillier, W. (2002). A brief overview of the relevance of Dabrowski's theory for the gifted. AGATE, 15, 4-13.

Tillier, W. (2008). Kazimierz Dabrowski: The man. In S. Mendaglio (Ed.), Dabrowski 's theory of positive disintegration (pp. 3-11). Scottsdale, AZ: Great Potential Press.

Tillier, W. (2013). The theory of positive disintegration by Kazimierz Dabrowski. Disponível em http://positivedisintegration.com. Acesso em 24 de maio de 2013.

Treat, A. R. (2006). Overexcitability in gifted sexually diverse populations. The Journal of Secondary Gifted Education, 17, 244-257.

Wirthwein, L., \& Rost, D. H. (2011). Focussing on overexcitabilities - studies with intellectually gifted and academically talented adults. Personality and Individual Differences, 51, 337-342.

Yakmaci-Guzel, B., \& Akarsu, F. (2006). Comparing overexcitabilities of gifted and non-gifted 10th grade students in Turkey. High Ability Studies, 17, 43-56.

Recebido em 19.11.2013

Primeira decisão editorial em 13.07.2015

Aceito em 13.07.2015 